\title{
Channel State-Aware Joint Dynamic Cell Coordination Scheme Using Adaptive Modulation and Variable Reuse Factor in OFDMA Downlink
}

\author{
Dae Wook Byun, Young Min Ki, and Dong Ku Kim \\ Yonsei University, Dept. of Electrical and Electronic Engineering \\ 134 Shinchon-Dong, Seodaemun-Gu, Seoul 120-749, Korea \\ \{ladiosop, mellow, dkkim\}@yonsei.ac.kr \\ http://mcl.yonsei.ac.kr
}

\begin{abstract}
In this paper, two different dynamic cell coordination stratagies for frequency selective and flat fading are proposed for efficient subcarrier allocation in the joint consideration of adaptive modulation and variable frequency reuse in the channel-aware OFDMA downlink multicellular environment. Compared to a conventional OFDMA system without cell coordination, where system throughput may become degraded due to the persistent interference from other cells, the proposed system dynamically allows RNC to apply different reuse factors on each subchannel and scheduling in consideration of channel and interference conditions of individual users so as to increase the system throughput and guarantee QoS of each user. In a selective fading channel, the proposed schemes showed 2.6 times as large throughput as that of a single reuse factor of one for all subcarriers. In a frequency flat fading, the dynamic scheme with the proposed scheduling achieves on average three times larger throughput than the conventional dynamic scheme [8].
\end{abstract}

\section{Introduction}

Future wireless communication system designs will require support for high data rates, provision of various quality of services (QoS) for multiple users, and operation in a multipath radio channel environment. Orthogonal frequency division multiple access (OFDMA) was proposed as one of the most promising technologies believed to satisfy most of these demands.

In either OFDMA or OFDM systems, one of the important issues is efficient subcarrier allocation to users. Some dynamic subcarrier allocation algorithms [1][3][4] were proposed for the multiple types of services that require various data rates. However, most of the algorithms were not considered in a multicell environment. An inter-cell interference avoidance technique was proposed [5]. A key consideration in designing a multicell cellular environment was frequency reuse, which is the ability to use the same frequencies repeatedly. A reuse scheme was proposed that divides a cell into several concentric zones in which 
each zone is assigned a different frequency reuse factor [6][7]. This scheme can be easily implemented but can not efficiently adapt time variation of mobile distribution and channels. Therefore, both frequency reuses and channel scheduling should be considered jointly for more efficient subcarrier allocation in a multicell environment.

Simplified Subchannel Allocation Scheme (SSAS) [8] were proposed by considering frequency reuse and adaptive modulation in the cellular OFDMA system in frequency flat fading. However, since its performance is degraded in practical environment priority based greedy schemes are proposed to enhance performance. In the case of frequency selective fading, the proposed cell coordination schemes achieves better performance than the static cell coordination scheme, considering different channel gain of each subchannel.

The remainder of the paper is organized as follows. In Chapter 2, the concept of frequency reuse and the system model are described, and the performance of the system using the single reuse factor is investigated. In Chapter 3, the dynamic cell coordination scheme [8] is reviewed and the proposed dynamic cell coordination schemes are introduced in frequency flat fading environment. In Chapter 4, novel dynamic cell coordination schemes are introduced in more detail in conjunction with frequency selective fading. In Chapter 5, the performance of the proposed schemes is demonstrated. Finally, conclusions are presented in Chapter 6 .

\section{System Model and No Cell Coordination}

\subsection{Reuse Factor and System Model}

A subchannel is defined as a group of adjacent subcarriers. The frequency reuse factor used in this paper is slightly different in that each cell of a cluster is allowed to access whole subchannels in the system and each subchannel of each cell can be assigned with different reuse factors. Hence, all subchannels in a cell are not always exploited in a cell unless the reuse factor of all subchannels is 1 .

Consider a downlink OFDMA system using adaptive modulation and coding (AMC) in a multicell environment of 37 hexagonal cells of $1 \mathrm{~km}$ radius for the reuse factors of 1,3 , and 7 . It is assumed that pilot signal contains base station (BS) index so that mobile station (MS) distinguishes pilots from each BS and estimates each different SINRs for corresponding reuse factors that could be used in the system. Each subchannel is assigned one of the reuse factors by schemes shown later on. The allocated power of each subchannel is assumed to be equal and the identical modulation scheme is applied to subcarriers within a subchannel. Parameters such as symbol duration and frequency offset are assumed to be designed such that inter-symbol interference and inter-channel interference can be neglected.

\subsection{No Cell Coordination with Single Reuse Factor}

The reuse factor of each subchannel is predetermined by the system. MS measures SINRs value and determines the transmittable data rates of the subchannels, 
which are transmitted to BS through feedback channel. BS receives the data rates of each subchannel from each MS and allocates subchannels according to the proportional fairness (PF) scheduling algorithm. The selection of users in the PF scheduling algorithm is well known as

$$
i_{n}^{*}=\arg \max _{i} \frac{R_{i, n}(t)}{\overline{R_{i, n}}(t)},
$$

where $\overline{R_{i, n}}(t)$ and $R_{i, n}(t)$ denote the average and the instantaneous data rates of the $n$-th subchannel of user $i$, respectively. PF scheduling is performed independently in each subchannel.

\subsection{The Performance in Using Single Reuse Factor}

1) Comparison of cell throughput: Fig. 1(a) illustrates the comparison of cell throughput for various reuse factors in frequency flat and frequency selective fading, where RF $k$ denotes reuse factor $k$. Among various reuse factors, it is easy to expect that the cell throughput for reuse factor of one is the largest. Cell throughput on frequency flat fading is higher than that of frequency selective fading, which has been shown in many other literatures.

2) Comparison of fairness: Fig. 1(b) illustrates the comparison of fairness for different reuse factors when the numbers of users are 5 and 15 , where it is easily expected that fairness performance is improved by using larger reuse factor.

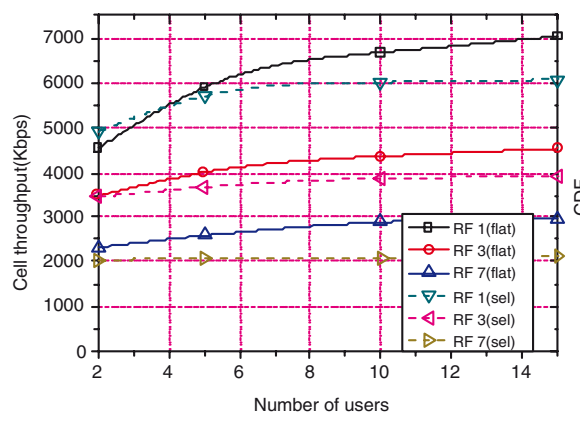

(a)

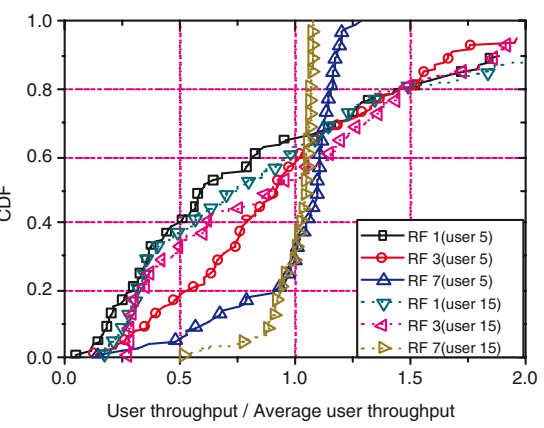

(b)

Fig. 1. (a) Cell throughput comparison in frequency flat and selective fading; (b) Comparison of fairness for reuse factors of 1,3 , and 7

\section{Dynamic Cell Coordination for Frequency Flat Fading}

\subsection{Static Cell Coordination}

In static cell coordination, radio network controller (RNC) determines the reuse factors of each subchannel, where the resultant reuse factors of each subchannel 
does not change throughout the transmission time. BS allocates the subchannel of certain reuse factors determined by RNC to users with a certain scheduling algorithm.

Only three different values of reuse factors, which are 1,3 , and 7 , are considered. The numbers of the subchannel of each reuse factor is denoted by $a / b / c$, where $\mathrm{a}$ is the number of subchannels of reuse factor of $1, \mathrm{~b}$ for 3 , and $\mathrm{c}$ for 7 . Three different frequency allocation, which are $(22 / 1 / 1),(9 / 3 / 2)$, and $(1 / 1 / 4)$, are considered, where $(22 / 1 / 1)$ is one using the reuse of one dominantly, $(9 / 3 / 2)$ for the reuse of three dominant, and $(1 / 1 / 4)$ for the reuse of seven dominant.

\subsection{Priority Based Greedy Cell Coordination Scheme}

\section{Priorities of Users}

1) The priority using spectral efficiency: A spectral efficiency of a subchannel is defined as ratio of transmittable data rate of user to reuse factor [8]. The priority of the user $i$ is computed by using spectral efficiency as follows:

$$
\text { Priority of user } i=\frac{R_{k}^{i b}}{k},
$$

where $R_{k}^{i b}$ is the achievable data rate of a subchannel with reuse factor $k$ for user $i$ in BS $b$. The highest priority is assigned to the user with the highest spectral efficiency per subchannel. Therefore, users having good channel condition are served more often, while those users having bad channel condition are served infrequently.

2) The priority using the spectral efficiency and the average user throughput: The priority of user is determined by considering $\bar{R}^{i b}$ as follows:

$$
\text { Priority of user } i=\left(\frac{R_{k}^{i b}}{k}\right) / \bar{R}^{i b} \text {, }
$$

where $\bar{R}^{i b}$ represents the average data rate of a subchannel for user $i$ in BS $b$. Unlike the case described by (2), (3) generates the spectral efficiency normalized by its average data rate, which is more fair than spectral efficiency itself.

\section{Priority Based Greedy Cell Coordination Schemes}

The proposed schemes offer three different ways of calculating the number of required subchannels of cells without changing function of RNC that was shown in SSAS [8], where the allocation of subchannels is done every frame in BS.

1) Priority based greedy cell coordination scheme 1 : According to the $R_{k}^{i b}$ of each user, the number of the required subchannels is obtained as follows:

$$
R_{k}^{i b} \cdot N_{k}^{i b} \geq T_{i}^{b}
$$

where $N_{k}^{i b}$ and $T_{i}^{b}$ denote the number of required subchannels and the predetermined required data rate for user $i$ in BS $b$, respectively. The scheme allocates subchannels in greedy manner while guaranteeing $T_{i}^{b}$ during every frame. 
2) Priority based greedy cell coordination scheme 2: It calculates $N_{k}^{i b}$ by using both average and the instantaneous data rate of user $i$ such that QoS does not need to satisfy $T_{i}^{b}$ within every frame but does in average sense. The number of the required subchannels of each user is computed as follows.

$$
\left(\frac{N-1}{N}\right) \cdot \bar{R}^{i b}+\frac{1}{N} \cdot R_{k}^{i b} \cdot N_{k}^{i b} \geq T_{i}^{b},
$$

where $N$ represents the moving window size used to evaluate the average data rate of user $i$.

3) Priority based greedy cell coordination scheme 3: It is basically similar to scheme 2 except that all surplus channels at each BS are assigned to the user with the highest priority.

\section{Cell Coordination for Frequency Selective Fading}

\subsection{Static Cell Coordination}

Static cell coordination in a selective fading is the same as in flat fading except that scheduling algorithm should be performed for each subchannel basis.

\subsection{Spectral Efficiency and Priority Based Dynamic Cell Coordination}

In the application of a frequency selective fading, in which channel gains are constant over a subchannel and independent of each subchannel, the amount of feedback information of channel condition to BS increases. An important observation is demonstration of the efficiency of the proposed coordination schemes with which subchannels are assigned multiple reuse factors to minimize intercell interference with priority of users.

Two schemes are proposed: the dynamic maximum $\mathrm{C} / \mathrm{I}$ cell coordination (DMCC) scheme and the dynamic proportional fairness cell coordination (DPFCC) scheme. In DMCC, subchannels are allocated to users by considering the spectral efficiency of users within each BS. In DPFCC, the spectral efficiency is replaced by the priority of users using an average user throughput. The proposed cell coordination schemes are composed of the following steps.

1) $M S$ report: MS measures three values of SINR assuming reuse factors of 1,3 , and 7 for each subchannel. The transmittable data rate at each subchannel is calculated from the measured SINRs and the corresponding spectral efficiencies are determined as follows.

$$
\begin{gathered}
e_{k, n}^{i b}=\frac{R_{k, n}^{i b}}{k} \\
k_{b e s t, n}^{i b}=\arg \max _{k} e_{k, n}^{i b},
\end{gathered}
$$


where $e_{k, n}^{i b}$ denotes the spectral efficiency of the $n$-th subchannel of user $i$ in BS $b$ with reuse factor of $k$. MS transmits $k_{\text {best,n }}^{i b}$ and $e_{k_{b e s t}, n}^{i b}$ values to BS.

2) BS report: In DMCC, the $b$-th BS gathers $k_{b e s t, n}^{i b}$ and $e_{k_{b e s t}, n}^{i b}$ values from users and creates the matrix $\mathbf{Q}^{b}$ which is formed by

$$
\mathbf{Q}^{b}=\left(\begin{array}{cccc}
e_{1,1}^{\hat{i} b} & e_{1,2}^{\hat{i} b} & \ldots & e_{1, n}^{\hat{i} b} \\
e_{3,1}^{\hat{i} b} & e_{3,2}^{\hat{i} b} & \ldots & e_{3, n}^{\hat{i} b} \\
e_{7,1}^{\hat{i} b} & e_{7,2}^{\hat{i} b} & \ldots & e_{7, n}^{\hat{i} b}
\end{array}\right)
$$

where $\hat{i}(k, n, b)=\arg \max _{i: \hat{k}=k} e_{\hat{k}, n}^{i b}$ and $\hat{i}$ denotes user who has the maximum spectral efficiency at the $n$-th subchannel with reuse factor $k$ in BS $b$. An element of the matrix $\mathbf{Q}^{b}$ has the largest spectral efficiency among the $n$-th subchannel of the same reuse factor of all users in BS. In DPFCC, the priority of users is evaluated as follows.

$$
\begin{aligned}
& p_{k_{\text {best }}, n}^{i b}=\frac{R_{k_{b e s t}, n}^{i b}}{\bar{R}^{i b}}
\end{aligned}
$$

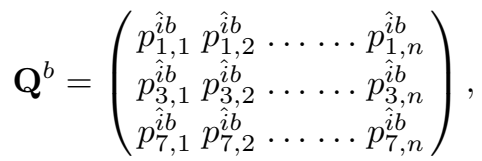

where $\hat{i}(k, n, b)=\arg \max _{i: \hat{k}=k} p_{\hat{k}, n}^{i b}$ and $\hat{i}$ denotes user who has the maximum priority at the $n$-th subchannel with reuse factor $k$ in $\mathrm{BS} b$.

The matrix $\mathbf{Q}_{\text {best }}^{b}$ is determined as follows:

$$
\mathbf{Q}_{b e s t}^{b}=\left(e_{k^{*}, 1}^{\hat{i}\left(k^{*}, n, b\right) b}, e_{k^{*}, 2}^{\hat{i}\left(k^{*}, n, b\right) b}, \cdots, e_{k^{*}, n}^{\hat{i}\left(k^{*}, n, b\right) b}\right),
$$

where $k^{*}(n, b)=\arg \max _{k} e_{k, n}^{\hat{i} b}$ and $k^{*}$ denotes reuse factor corresponding to the maximum value among spectral efficiencies of $n$-th subchannel in $\mathrm{BS} b . k^{*}(n, b)$, $\mathbf{Q}_{\text {best }}^{b}$, and $\mathbf{Q}^{b}$ evaluated at each BS are sent to RNC.

3) Cell coordination: RNC executes cell coordination as follows.

Step 1. Reuse factor Determination for each subchannel: The number of reuse factors requested from all BS calculations in each subchannel are counted. A nominal reuse factor in each subchannel is determined one that receives the largest request from all BSs in order to use efficiently available system bandwidth. For example, if the requested number of reuse factors of 1,3 , and 7 at a certain subchannel gathered from all BSs are 9, 9, and 1, respectively, the nominal reuse factor in the subchannel is determined to be 3 . Once the nominal reuse factor is fixed to be $k$ in a subchannel, then the nominal spectral efficiency is determined as the largest one among the spectral efficiencies of those BS having requested reuse factor of $k$. 
Step 2. Band partitioning for reuse factor seven: Different band partitioning methods can exist and one of them was implemented below. We need to compare the performance and the complexity with respect to different band partitioning ways from now on.

The proposed schemes determine the band of frequency reuse factors of $k$ by moving subband windows covering 7 consecutive subchannels over the entire band. In each move, the number of times a reuse factor of 7 occurred is counted and the spectral efficiencies of subchannels exhibiting a reuse factor of 7 are summed to represent the spectral efficiency of the subband. Windows having more than four subchannels of reuse factor 7 are assigned as candidate subbands of reuse factor seven. Subsequently, subband window of size seven is moved right by one subchannel and the procedure is repeated until the subband window of size seven covers all subchannels. In order to determine the subband of reuse factor 7 among the candidates, the sums of spectral efficiencies of subbands are sorted by descending order. If two subbands are overlapped, the subband having the smaller sum is removed.

Step 3. Band partitioning for reuse factor three: Step 2 is repeated for subbands having a reuse factor of 3 . In this instance, subband windows having more than two subchannels of reuse factor of 3 become candidates for the category.

Step 4. Band partitioning for reuse factor one: After steps 2 and 3 are conducted for reuse factors 7 and 3 , all remaining subchannels that are not yet determined are assigned a reuse factor equal to $1 . k_{n}$, which represents the assigned reuse factors for each subchannel, is thereby generated in this step.

Step 5. BS determination for each subchannel: For subbands of reuse factor 7 , $\mathrm{RNC}$ allocates each subchannel to the BS having the largest spectral efficiencies among seven BSs. If two or more subchannels are assigned to the same BS, RNC allows BS to select only one subchannel having the largest spectral efficiency and repeats subchannel allocation for the those subchannels that have yet to be assigned to BS. The identical method is used to allocate subchannels of reuse factor 3 to each $\mathrm{BS}$. The matrix $\mathbf{B}$, indicating which $\mathrm{BS}$ uses a certain subchannel, is determined as follows:

$$
\mathbf{B}=\left(b_{0}, b_{1}, \cdots, 0, \cdots, b_{n}\right),
$$

where $b_{n}$ is the BS index of the $n$-th subchannel and $b_{n}=0$ denotes that the reuse factor of the $n$-th subchannel is 1 .

Step 6. Subchannel allocation to $B S$ : RNC transmits $k_{n}$ and the matrix $\mathbf{B}$ to BSs.

4) Allocating subchannel to $M S$ : Each BS receives the information about the subchannels to be used and the reuse factors assigned. BS allocates each subchannel to user with highest priority during one downlink frame using the matrixes $\mathbf{Q}^{b}$, $\mathbf{B}$, and the value for $k_{n}$. 


\section{Simulation Results}

\subsection{Simulation Environments}

Table 1 shows parameters of IEEE 802.16e-based TDD-OFDMA system. The MCS level [13][14] is reported to users according to SINR sensitivity thresholds and the delay of one frame is assumed in MCS feedback. The existence of 37 cells having a $1 \mathrm{~km}$ cell radius is assumed. The required data rate is assigned to be the same for all users. MSs are distributed uniformly in BSs and the number of MSs per cell is assumed from 2 to 15. The path loss model is assumed to be $P L=129.427+37.6 * \log _{10}\left(d_{k m}\right)[9][10]$ and the standard deviation of log-normal shadowing is $10 \mathrm{~dB}$. Short-term channel gains are assumed to be Rayleigh fading with a Doppler frequency of $6.4 \mathrm{~Hz}$ and the tapped-delay-line multipath models from ITU-R were used [11]. The BS transmitted power and antenna gains were set to values of $20 \mathrm{~W}$ and $14 \mathrm{dBi}$, respectively. The thermal noise density was assumed to be $-174 \mathrm{dBm} / \mathrm{Hz}$ and the maximum $\mathrm{C} / \mathrm{I}$ value was limited to 30 $\mathrm{dB}$. In all performance figures, the notations of sp and nsp denote the spectral efficiency and the spectral efficiency normalized by the average throughput.

Table 1. System parameters [12]

\begin{tabular}{cc}
\hline Parameters & Value \\
\hline Carrier Frequency & $2.3 \mathrm{GHz}$ \\
Channel Bandwidth & $10 \mathrm{MHz}$ \\
Number of subcarriers & 1,702 of 2,048 \\
Number of traffic subcarriers & 1,536 \\
Subcarrier spacing & $5.57617 \mathrm{kHz}$ \\
Number of subchannels & 32 \\
Number of subcarriers & 48 \\
Frame length & $5.0 \mathrm{msec}$ \\
Number of DL symbols & 18 \\
OFDMA symbol time & $190.543 \mu \mathrm{sec}$ \\
Guard interval & $11.208 \mu \mathrm{sec}$ \\
\hline
\end{tabular}

\subsection{Cell Throughput Performance}

The cell throughput of the static and dynamic cell coordination schemes for the various numbers of users in frequency flat fading are shown in Fig. 2 and 3. Cell throughput using SSAS [8] decreases as the number of users increases to greater than five because of the increased likelihood of more users requesting more subchannels of reuse factor 7 . When the target data rate is $384 \mathrm{kbps}$ and the number of users becomes larger, the cell throughput of greedy cell coordination scheme 2 outperforms SSAS by a maximum of $66 \%$. In the case of $64 \mathrm{kbps}$, the greedy cell coordination scheme 3 achieves an average of 3.6 times greater cell throughput compared to SSAS. The performance of greedy scheme 3 is 


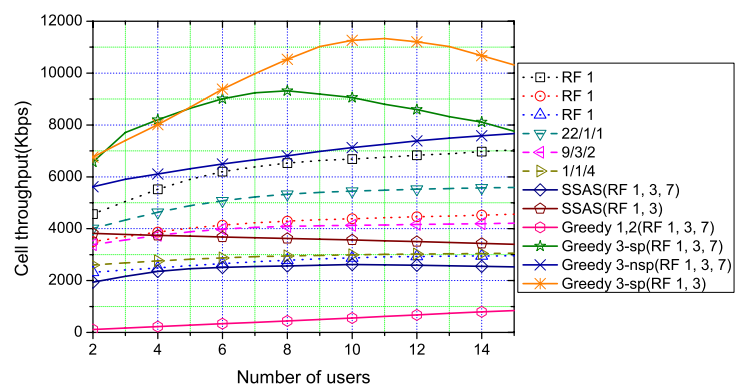

Fig. 2. Cell throughput comparison for the static approach, SSAS, and the schemes proposed for a data rate of $64 \mathrm{kbps}$ in frequency flat fading

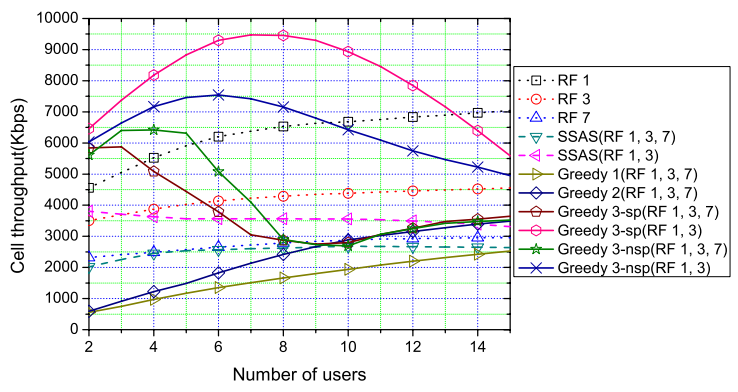

Fig. 3. Cell throughput comparison of SSAS and the schemes proposed for a required data rate of $384 \mathrm{kbps}$ in frequency flat fading

degraded as the number of users increases since fewer users are allocated the required throughput when the required data rate of users is $384 \mathrm{kbps}$. The cell throughput of greedy scheme 3 using reuse factors equal to 1 and 3 is decreased as compared to applying the scheme using reuse factors 1,3 , and 7 .

The cell throughput comparison of various cell coordination systems including no coordination and dynamic coordination within a range of the number of users in frequency selective fading is illustrated in Fig. 4. DMCC demonstrates the best cell throughput because it allocates subchannels to users having good channel condition. The cell throughput of DMCC analysis using all reuse factors of 1,3 , and 7 is about 2.6 times greater than that for the scheme using a reuse factor of only 1. DPFCC exhibit relatively low throughput as compared to DMCC because the fairness among users is considered. Nevertheless, the cell throughput is similar to that of the case using the single reuse factor of 3 as the number of users increases. Also, the DPFCC using reuse factor 1 and 3 approaches to the cell throughput of the case of single reuse factor 1 . DMCC and DPFCC calculations using reuse factor values of 1 and 3 demonstrate $24 \%$ and $57 \%$ more 


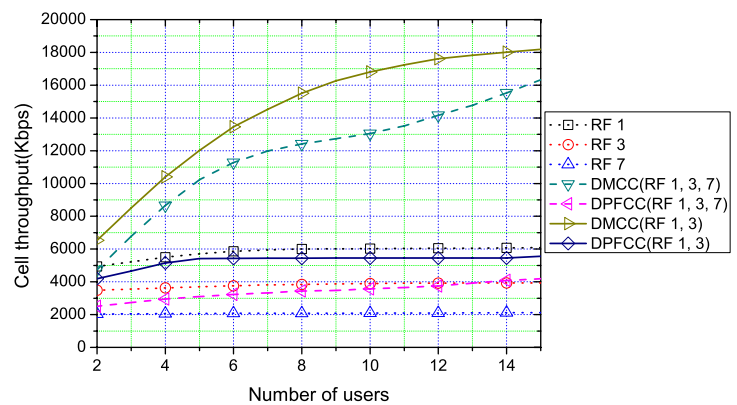

Fig. 4. Cell throughput comparison of schemes proposed using reuse factor values of 1, 3 and 7 or reuse factor values of 1 and 3 in frequency selective fading

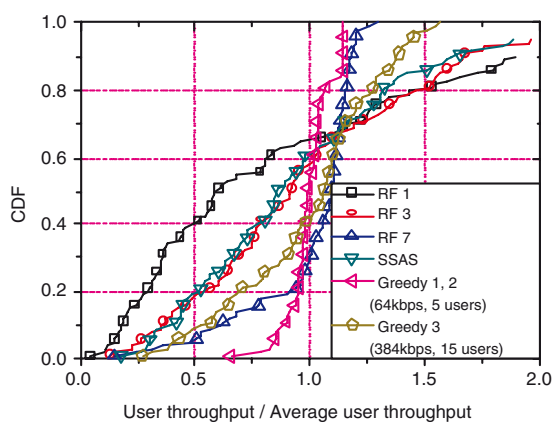

(a)

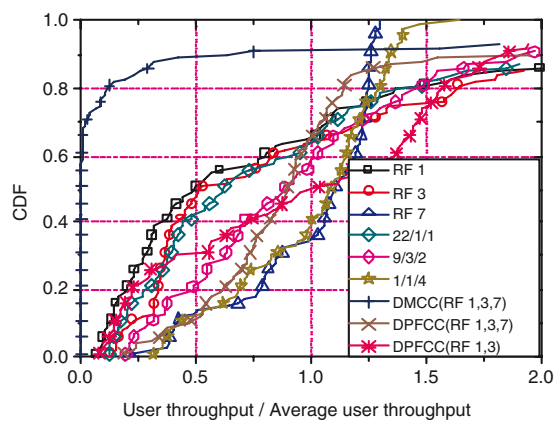

(b)

Fig. 5. (a) Fairness comparison of SSAS and the proposed schemes in flat fading; (b) Fairness comparison of proposed schemes in selective fading

cell throughput, respectively, compared to calculations using all reuse factor values of 1, 3 and 7 because the subchannel with reuse factor 7 used one seventh of the total subchannels available.

\subsection{Fairness Performance}

The relative fairness of SSAS and the schemes proposed in this research were compared for frequency flat fading, given that the numbers of users are 5 and 15 and the required data rates of users are $64 \mathrm{kbps}$ and $384 \mathrm{kbps}$, respectively in Fig. 5(a). The fairness of SSAS is similar to the case using the single reuse factor equal to 3 and is independent of the number of users and the required data rate of users. When the required data rate of users is $64 \mathrm{kbps}$, the greedy scheme 1 and 2 demonstrate better fairness than the case using a reuse factor of 7 . As the required data rate for 15 users was $384 \mathrm{kbps}$, the fairness of greedy scheme 3 approach to the case of $64 \mathrm{kbps}$ using greedy schemes 1 and 2 because there are hardly the remaining subchannels. 
The fairness comparison of the proposed schemes assuming 15 users in frequency selective fading was also assessed in Fig. 5(b). The fairness values of $(22 / 1 / 1)$ and $(1 / 1 / 4)$ approach to that for single reuse factors equal to 1 and 3 , respectively. DMCC demonstrate the worst fairness because it allocates subchannels to the users having the best channel quality. By using DMCC, $65 \%$ of users are not served while the fairness is degraded. The fairness assessment of DPFCC using all reuse factors of 1,3 , and 7 achieve better performance than that of $(9 / 3 / 2)$. The fairness level of DPFCC using reuse factors 1 and 3 is poorer than that of DPFCC using reuse factors of 1,3 , and 7 . In flat and selective fading environment, it is observed that most of cell coordination schemes could offer data service to users in the cell boundary by improving their SINRs using large reuse factors.

\section{Concluding Remarks}

Two different dynamic cell coordination schemes considering jointly adaptive modulation and variable frequency reuse were proposed to allocate subcarrier efficiently in the channel condition aware OFDMA downlink multicell system. The performance was evaluated in frequency flat and selective fading. The proposed system dynamically allows RNC to apply different reuse factors on each subchannel and scheduling in consideration of channel and interference conditions of individual users so as to increase the system throughput and guarantee QoS of each user. In frequency flat fading, for the required data rate of $64 \mathrm{kbps}$, it was demonstrated that greedy scheme 3 achieved on average a 3.6 times higher cell throughput as compared to SSAS and that greedy schemes 1 and 2 produced the best performance of fairness. At the required data rate of $384 \mathrm{kbps}$, the cell throughput of greedy scheme 3 was, at the maximum, 3 times higher than that of SSAS. Greedy scheme 3 approached to the case of $64 \mathrm{kbps}$ using greedy schemes 1 and 2. In frequency selective fading, the cell throughput of DMCC was, at the maximum, 2.6 times higher than that of the case using a reuse factor of 1 , and the fairness of DPFCC approached that when the reuse factor equal to 7 was applied.

\section{Acknowledgment}

This work was supported by the Korea Research Foundation Grant (KRF-2004013-D00060).

\section{References}

1. Kivanc D., Li G., Liu H.: Computationally Efficient Bandwidth Allocation and Power Control for OFDMA. IEEE Transsaction on Wireless Communications, Vol. 2. IEEE. (2003) 1150-1158.

2. Tse D.: Multiuser diversity in wireless networks. Wireless communication seminar, (2001). 
3. Wong C. Y., Cheng R. S.: Multiuser OFDM with Adaptive Subcarrier, Bit, and Power Allocation. IEEE Journal on Selected Areas in Communication, Vol. 17. IEEE. (1999) 1747-1758.

4. Kivanc D., Liu H.: Subcarrier Allocation and Power Control for OFDMA. Signals, Systems and Computers, Conference Record of the Thirty-Fourth Asilomar Conference on, Vol. 1. (2000) 147-151.

5. Suzuki M., Bohnke R., Sakoda K.: Band division multiple access (BDMA) system: A novel approach for next generation mobile telecommunication system, based on OFDM and SFH-TDMA. IEEE Vehicular Technology Conference (VTC 1998).

6. Zander J., Frodigh M.: Capacity allocation and channel assignment in cellular radio systems using reuse partitioning. Electronics Letters, Vol. 28. (1992) 438-440.

7. Blair P., Polyzos G. C., Zorzi M.: Plane Cover Multiple Access: A New Approach to Maximizing Cellular System Capacity. IEEE Journal on Selected Areas in Communication, Vol. 19. IEEE. (2001) 2131-2141.

8. Kim H., Han Y., Koo J.: Optimal Subchannel Allocation Scheme in Multicell OFDMA Systems, IEEE Vehicular Technology Conference (VTC 2004) 1821-1825.

9. Recommendation ITU-R M.1225, Guideline for Evaluation of Radio Transmission Technologies for IMT-2000, (1997).

10. TTAR-0016, Evaluation Criteria of Radio Access Technology for 2.3GHz Portable Internet, Telecommunications Technology Association (TTA), (2004).

11. 3GPP R1-030042, Update of OFDM SI simulation methodology. (2003).

12. http://www.ieee802.org/16/tge

13. IEEE C802.16d-03/78r1, Coverage/Capacity simulation for OFDMA PHY in with ITU-T channel model, (2003).

14. IEEE C802.16d-04/50r3, OFDMA PHY Enhancements for better mobility performance, (2004). 